\title{
Minimal Length Schrödinger Equation with Harmonic Potential in the Presence of a Magnetic Field
}

\author{
H. Hassanabadi, ${ }^{1}$ E. Maghsoodi, ${ }^{2}$ Akpan N. Ikot, ${ }^{3}$ and S. Zarrinkamar ${ }^{4}$ \\ ${ }^{1}$ Department of Physics, Shahrood University, Shahrood, Iran \\ ${ }^{2}$ Department of Basic Sciences, Shahrood Branch, Islamic Azad University, Shahrood, Iran \\ ${ }^{3}$ Theoretical Physics Group, Department of Physics, University of Uyo, Nigeria \\ ${ }^{4}$ Department of Basic Sciences, Garmsar Branch, Islamic Azad University, Garmsar, Iran
}

Correspondence should be addressed to E. Maghsoodi; e.maghsoodil84@gmail.com

Received 18 August 2013; Revised 20 October 2013; Accepted 20 October 2013

Academic Editor: Elias C. Vagenas

Copyright (C) 2013 H. Hassanabadi et al. This is an open access article distributed under the Creative Commons Attribution License, which permits unrestricted use, distribution, and reproduction in any medium, provided the original work is properly cited.

\begin{abstract}
Minimal length Schrödinger equation is investigated for harmonic potential in the presence of magnetic field and illustrates the wave functions in the momentum space. The energy eigenvalues are reported and the corresponding wave functions are calculated in terms of hypergeometric functions.
\end{abstract}

\section{Introduction}

The study of theories characterized by a minimal observable length is an active area of research in theoretical physics, not only because of their intrinsic interest, but also because of their existence as suggested by string theory and quantum gravity. In order to incorporate the idea of the minimal length into quantum mechanics, we need to modify the ordinary uncertainty principle to the so-called Generalized Uncertainty Principle (GUP). Some authors have worked on generalized quantum theoretical framework [1-16]. Scardigli has studied a version of GU4 obtained in string theory and in gedanken experiments carried out in quantum gravity [1]. In [4], Dadić et al. [4] have investigated deformed harmonic oscillators with minimal length uncertainty relations. Chang et al. [5] have determined the energy eigenvalues and eigenfunctions of the harmonic oscillator in arbitrary dimensions with minimal length uncertainty relations in [5]. The authors have considered the effects of the deformation on classical limit of the minimal length uncertainty relation in a central force potential in [6]. Recently much attention has been devoted to the study of deformed Heisenberg algebras of different kinds with minimal length [17-21]. For example, in [17], Ali and Tawfik [17] have studied the effects of GUP, which is proposed by some approaches to quantum gravity such as string theory, black hole physics, and doubly special relativity theories (DSR), on the area law of the entropy. The authors in [18] have solved the minimal length Schrödinger equation in the presence of a nonminimal Woods-Saxon interaction. The solution of the relativistic and nonrelativistic fundamental dynamical equations is an interesting phenomenon in many fields of physics. Recently, the analytic exact solutions of the nonrelativistic equation with some potential by different method are impossible [2228]. Many other aspects of the theory are well addressed in [29-35] and references therein. The interface of the minimal length quantum mechanics with quantum gravity is discussed in [29]. The application of the formalism to the motion of a dipole in a cosmic string background was studied in [30]. Chang et al. commented on the minimal length uncertainty relation and the essentials of string theory [31]. Harbach and his coworkers proposed the precision muon tests to investigate the minimal length scale [32]. Kothawala et al. studied the problem for space times with constant curvatures [33]. Panella considered the Casimir-Polder intermolecular forces within the framework of minimal length theories [34]. Pedram et al. analyzed the effects of minimal length on the transition rate of ultracold neutrons in the gravitational field [35]. Our work is organized as follows. In Section 2, we give a brief introduction of the Generalized Uncertainty 
Principle. In Section 3, the Schrödinger equation is written in the momentum space including the harmonic potential and it obtains the solution of the problem. We use the useful analytical Nikiforov-Uvarov technique in our calculations (Appendix A). Finally, the conclusion is given in Section 4.

\section{The Generalized Uncertainty Principle}

An immediate consequence of the minimal length is the Generalized Uncertainty Principle (GUP) [7]

$$
\Delta x \geq \frac{\hbar}{\Delta p}+\alpha l_{p}^{2} \frac{\Delta p}{\hbar},
$$

where the GUP parameter $\alpha$ is determined from a fundamental theory [7]. At low energies, that is, energies much smaller than the Planck mass, the second term in the right hand side of the latter vanishes and we recover the wellknown Heisenberg uncertainty principle. The GUP of (1) corresponds to the generalized commutation relation [7]

$$
\left[x_{o p}, p_{o p}\right]=i \hbar\left(1+\beta p^{2}\right), \quad 0 \leq \beta \leq 1,
$$

where $x_{o p}=\widehat{x}$ and $0 \leq \beta \leq 1$. The limits $\beta \rightarrow 0$ and $\beta \rightarrow$ 1 , respectively, correspond to the normal quantum mechanics and extreme quantum gravity. Equation (2) gives the minimal length in this case as $(\Delta x)_{\min }=2 l_{p} \sqrt{\alpha}$ and a representation of $\widehat{x}_{i}$ and $\widehat{p}_{i}$ in momentum space is taken as (Appendix B)

$$
\widehat{x}_{i}=i \hbar\left(1+\beta p^{2}\right) \frac{\partial}{\partial p_{i}}, \quad \widehat{p}_{i}=p_{i}
$$

\section{The Equation in the Momentum Space}

The minimal length Schrödinger equation in momentum space $[\Psi(\vec{r}) \rightarrow \Phi(\vec{p})]$ is

$$
\left\{\frac{\Pi^{2}}{2 m}+V(r)\right\} \Phi_{n \lambda}(\vec{p})=E_{n \lambda} \Phi_{n \lambda}(\vec{p}),
$$

where $E, \vec{\Pi}$, and $m$, respectively, denote the energy of the system, two-dimensional momentum operator, and the mass of the particle. By considering $\vec{\Pi}=\vec{p}-(e / c) \vec{A}$, the minimal length Schrödinger equation appears as

$$
\left\{\frac{(\vec{p}-(e / c) \vec{A})^{2}}{2 m}+V(r)\right\} \Phi_{n \lambda}(\vec{p})=E_{n \lambda} \Phi_{n \lambda}(\vec{p}),
$$

where the vector potential $\vec{A}$ can be alternatively expressed as $A=(-B \widehat{y} / 2, B \widehat{x} / 2,0)$ and $\vec{B}=B \widehat{z}$ is the applied magnetic field. We consider the following harmonic potential:

$$
V(r)=V_{0} r^{2}
$$

Now, by inserting the latter into (5), we have

$$
\begin{aligned}
& \left\{\frac{\left[\left(p_{x}-(e / c) A_{x}\right) \hat{i}+\left(p_{y}-(e / c) A_{y}\right) \hat{j}\right]^{2}}{2 m}+V_{0}\left(\widehat{x}^{2}+\widehat{y}^{2}\right)\right\} \\
& \times \Phi_{n \lambda}(\vec{p})=E_{n \lambda} \Phi_{n \lambda}(\vec{p}) .
\end{aligned}
$$

A representation of $\widehat{x}$ and $\widehat{y}$ is taken as

$$
\begin{aligned}
& \widehat{x}=i\left(1+\beta p^{2}\right) \frac{\partial}{\partial p_{x}}, \\
& \widehat{y}=i\left(1+\beta p^{2}\right) \frac{\partial}{\partial p_{y}},
\end{aligned}
$$

where we have defined $p_{x}=p \cos \theta$ and $p_{y}=p \sin \theta$. Substitution of (8a) and (8b) into (7) gives

$$
\begin{aligned}
\left\{\frac{1}{2 m}[\right. & \left(p_{x}+\frac{e}{c} \frac{B}{2} i\left(1+\beta p^{2}\right) \frac{\partial}{\partial p_{y}}\right) \\
& \times\left(p_{x}+\frac{e}{c} \frac{B}{2} i\left(1+\beta p^{2}\right) \frac{\partial}{\partial p_{y}}\right) \\
+ & \left(p_{y}-\frac{e}{c} \frac{B}{2} i\left(1+\beta p^{2}\right) \frac{\partial}{\partial p_{x}}\right) \\
\times & \left.\left(p_{y}-\frac{e}{c} \frac{B}{2} i\left(1+\beta p^{2}\right) \frac{\partial}{\partial p_{x}}\right)\right] \\
+V_{0}[ & \left(i\left(1+\beta p^{2}\right) \frac{\partial}{\partial p_{x}}\right)\left(i\left(1+\beta p^{2}\right) \frac{\partial}{\partial p_{x}}\right) \\
& \left.+\left(i\left(1+\beta p^{2}\right) \frac{\partial}{\partial p_{y}}\right)\left(i\left(1+\beta p^{2}\right) \frac{\partial}{\partial p_{y}}\right)\right] \\
\left.-E_{n \lambda}\right\} & \Phi_{n \lambda}(\vec{p})=0 .
\end{aligned}
$$

Consider

$$
\begin{gathered}
\frac{\partial}{\partial p_{x}}=\cos \vartheta \frac{\partial}{\partial p}-\frac{\sin \vartheta}{p} \frac{\partial}{\partial \vartheta} \\
\frac{\partial}{\partial p_{y}}=\sin \vartheta \frac{\partial}{\partial p}+\frac{\cos \vartheta}{p} \frac{\partial}{\partial \vartheta} .
\end{gathered}
$$

Equation (9) then takes the form

$$
\begin{gathered}
\left\{\frac { 1 } { 2 m } \left[p^{2}+\frac{e}{c} \frac{B}{2} i\left(1+\beta p^{2}\right) \frac{\partial}{\partial \vartheta}-\frac{e^{2} B^{2}}{2 c^{2}} \beta\left(1+\beta p^{2}\right) p \frac{\partial}{\partial p}\right.\right. \\
-\frac{e^{2} B^{2}}{4 c^{2}}\left(1+\beta p^{2}\right)^{2} \frac{\partial^{2}}{\partial p^{2}}-\frac{e^{2} B^{2}}{4 c^{2}}\left(1+\beta p^{2}\right)^{2} \frac{1}{p} \frac{\partial}{\partial p} \\
\left.-\frac{e^{2} B^{2}}{4 c^{2}}\left(1+\beta p^{2}\right)^{2} \frac{1}{p^{2}} \frac{\partial^{2}}{\partial \vartheta^{2}}\right] \\
+V_{0}\left[-2 \beta\left(1+\beta p^{2}\right) p \frac{\partial}{\partial p}\right. \\
-\left(1+\beta p^{2}\right)^{2} \frac{\partial^{2}}{\partial p^{2}}-\left(1+\beta p^{2}\right)^{2} \frac{1}{p} \frac{\partial}{\partial p} \\
\left.\left.-\left(1+\beta p^{2}\right)^{2} \frac{1}{p^{2}} \frac{\partial^{2}}{\partial \vartheta^{2}}\right]-E_{n \lambda}\right\} \Phi_{n \lambda}(\vec{p})=0 .
\end{gathered}
$$


By using the solution of the form,

$$
\Phi_{n \lambda}(\vec{p})=\phi_{n \lambda}(p) e^{i \lambda \vartheta}
$$

reduces the above equation to the form

$$
\begin{aligned}
& \left\{\left[-\frac{e^{2} B^{2}}{8 m c^{2}}\left(1+\beta p^{2}\right)^{2}-V_{0}\left(1+\beta p^{2}\right)^{2}\right] \frac{\partial^{2}}{\partial p^{2}}\right. \\
& +\left[-\frac{e^{2} B^{2}}{4 m c^{2}} \beta\left(1+\beta p^{2}\right) p-\frac{e^{2} B^{2}}{8 m c^{2}}\left(1+\beta p^{2}\right)^{2} \frac{1}{p}\right. \\
& \left.\quad-2 \beta V_{0}\left(1+\beta p^{2}\right) p-V_{0}\left(1+\beta p^{2}\right)^{2} \frac{1}{p}\right] \frac{\partial}{\partial p} \\
& +\frac{p^{2}}{2 m}-\frac{e B}{4 m c} \lambda\left(1+\beta p^{2}\right)+\frac{e^{2} B^{2}}{8 m c^{2}}\left(1+\beta p^{2}\right)^{2} \frac{\lambda^{2}}{p^{2}} \\
& \left.+V_{0}\left(1+\beta p^{2}\right)^{2} \frac{\lambda^{2}}{p^{2}}-E_{n \lambda}\right\} \phi_{n \lambda}(\vec{p})=0 .
\end{aligned}
$$

In order to obtain the solution of (13), we use the transformation $s=-\beta p^{2}$ and rewrite it as follows:

$$
\begin{aligned}
& \frac{d^{2} \phi_{n \lambda}(s)}{d s^{2}}+\frac{(\eta-(\eta-\gamma) s)}{s(1-s)} \frac{d \phi_{n \lambda}(s)}{d s} \\
& +\frac{1}{s^{2}(1-s)^{2}}\left(\sigma s^{2}+\tau s+\rho\right) \phi_{n \lambda}(s)=0,
\end{aligned}
$$

where

$$
\begin{gathered}
\eta=\frac{1}{2}-\frac{e^{2}}{2 m c^{2}} \frac{B^{2}}{8 \mu}-\frac{V_{0}}{2 \mu}, \\
\gamma=\frac{e^{2}}{2 m c^{2}} \frac{B^{2}}{4 \mu}+\frac{V_{0}}{\mu}, \\
\mu=-\frac{e^{2}}{2 m c^{2}} \frac{B^{2}}{4}-V_{0}, \\
\sigma=\frac{1}{8 m \beta^{2} \mu}-\frac{e}{2 m c} \frac{B \lambda}{8 \beta \mu}+\frac{e^{2}}{2 m c^{2}} \frac{B^{2} \lambda^{2}}{16 \mu}+\frac{V_{0} \lambda^{2}}{4 \mu}+E_{n \lambda}, \\
\tau=\frac{e}{2 m c} \frac{B \lambda}{8 \beta \mu}-\frac{e^{2}}{2 m c^{2}} \frac{B^{2} \lambda^{2}}{8 \mu}-\frac{V_{0} \lambda^{2}}{2 \mu}-E_{n \lambda}, \\
\rho=\frac{e^{2}}{2 m c^{2}} \frac{B^{2} \lambda^{2}}{16 \mu}+\frac{V_{0} \lambda^{2}}{4 \mu} .
\end{gathered}
$$

By comparing (14) with (A.11), we get

$$
\begin{aligned}
& \alpha_{1}=\eta, \quad \alpha_{2}=\eta-\gamma, \quad \alpha_{3}=1, \\
& \xi_{1}=-\sigma, \quad \xi_{2}=\tau, \quad \xi_{3}=-\rho, \\
& \alpha_{4}=\frac{1}{2}(1-\eta), \quad \alpha_{5}=\frac{\eta-\gamma}{2}-1, \\
& \alpha_{6}=\left(\frac{\eta-\gamma}{2}-1\right)^{2}-\sigma, \\
& \alpha_{7}=\left(\frac{(1-\eta)(\eta-\gamma)}{2}-(1-\eta)\right)-\tau \text {, } \\
& \alpha_{8}=\frac{1}{4}(1-\eta)^{2}-\rho, \\
& \alpha_{9}=\left(\frac{(1-\eta)(\eta-\gamma)}{2}-(1-\eta)\right)-\tau+\frac{1}{4}(1-\eta)^{2} \\
& -\rho+\left(\frac{\eta-\gamma}{2}-1\right)^{2}-\sigma \\
& =\frac{\gamma^{2}}{4}+\frac{\gamma}{2}+\frac{1}{4}-\tau-\rho-\sigma, \\
& \alpha_{10}=1+2 \sqrt{\frac{1}{4}(1-\eta)^{2}-\rho}, \\
& \alpha_{11}=2+2\left(\sqrt{\frac{\gamma^{2}}{4}+\frac{\gamma}{2}+\frac{1}{4}-\tau-\rho-\sigma}\right. \\
& \left.+\sqrt{\frac{1}{4}(1-\eta)^{2}-\rho}\right) \\
& \alpha_{12}=\frac{(1-\eta)}{2}+\sqrt{\frac{1}{4}(1-\eta)^{2}-\rho}, \\
& \alpha_{13}=\frac{\eta-\gamma}{2}-1-\left(\sqrt{\frac{\gamma^{2}}{4}+\frac{\gamma}{2}+\frac{1}{4}-\tau-\rho-\sigma}\right. \\
& \left.+\sqrt{\frac{1}{4}(1-\eta)^{2}-\rho}\right) .
\end{aligned}
$$

From (A.20), we have

$$
\begin{gathered}
n^{2}+n+\frac{1}{2}+\frac{\eta \gamma}{2}+(2 n+1)\left(\sqrt{\frac{\gamma^{2}}{4}+\frac{\gamma}{2}+\frac{1}{4}-\tau-\rho-\sigma}\right. \\
+\sqrt{\left.\frac{1}{4}(1-\eta)^{2}-\rho\right)}-\tau-2 \rho \\
+2 \sqrt{\left(\frac{1}{4}(1-\eta)^{2}-\rho\right)\left(\frac{\gamma^{2}}{4}+\frac{\gamma}{2}+\frac{1}{4}-\tau-\rho-\sigma\right)}=0 .
\end{gathered}
$$


For this case, from (A.24), the wave function is

$$
\begin{aligned}
\phi_{n \lambda}(p)= & \left(-\beta p^{2}\right)^{(1-\eta) / 2+\sqrt{(1 / 4)(1-\eta)^{2}-\rho}} \\
& \times\left(1+\beta p^{2}\right)^{(\gamma+1) / 2+\left(\sqrt{\gamma^{2} / 4+\gamma / 2+1 / 4-\tau-\rho-\sigma}\right)} \\
& \times P_{n}^{\left(2 \sqrt{(1 / 4)(1-\eta)^{2}-\rho}, 2 \sqrt{\gamma^{2} / 4+\gamma / 2+1 / 4-\tau-\rho-\sigma}\right)} \\
& \times\left(1+2 \beta p^{2}\right),
\end{aligned}
$$

where $p_{n}^{(\alpha, \beta)}(1-2 z)$ is the Jacobi polynomial or

$$
\begin{aligned}
P_{n}^{(\alpha, \beta)} & (1-2 z) \\
& =\frac{1}{n !} z^{-\alpha}(1-z)^{-\beta} \frac{d^{n}}{d z^{n}}\left[z^{n+\alpha}(1-z)^{n+\beta}\right] .
\end{aligned}
$$

Therefore, $\Phi_{n \lambda}(\vec{p})$ can be written versus the hypergeometric function as

$$
\begin{aligned}
& \Phi_{n \lambda}(\vec{p})=\left(-\beta p^{2}\right)^{(1-\eta) / 2+\sqrt{(1 / 4)(1-\eta)^{2}-\rho}} \\
& \times\left(1+\beta p^{2}\right)^{(\gamma+1) / 2+\left(\sqrt{\gamma^{2} / 4+\gamma / 2+1 / 4-\tau-\rho-\sigma}\right)} \\
& \times{ }_{2} F_{1}\left(-n, n+2 \sqrt{\frac{1}{4}(1-\eta)^{2}-\rho}\right. \\
&+2 \sqrt{\frac{\gamma^{2}}{4}+\frac{\gamma}{2}+\frac{1}{4}}-\tau-\rho-\sigma+1 \\
&\left.2 \sqrt{\frac{1}{4}(1-\eta)^{2}-\rho}+1 ;-\beta p^{2}\right) e^{i \lambda \vartheta} .
\end{aligned}
$$

\section{Conclusion}

Exact solution of the Schrödinger equation of harmonic oscillator in a magnetic field considered in the presence of a minimal length has been obtained in terms of hypergeometric functions. We have reported eigenfunction and eigenvalues of energy. Having found the wavefunction and the spectrum, we can simply obtain the decay rate and the bounds on the minimal length parameter. In addition, many other important quantities in various fields of physics can be systematically obtained including the charge radius, form factors, and shifts in the Landau levels. More generally, we know that the minimal length formulation is in fact a parallel approach to the noncommutative formulation of the quantum theory. Therefore, roughly speaking, the whole story about the experimental verification of the noncommutative version of quantum mechanics (including experiments with $\mathrm{TeV}$ photon experiments, very precise measurement of the radiation emitted during the transition $1 S-2 S$ of the hydrogen atom, quantum optics, and Lamb shift studies) can be used here to comment on the results as well.

\section{Appendices}

\section{A.}

A.1. The Nikiforov-Uvarov Method. In this section, the NU method and its parametric generalization are briefly presented. The NU method, in its general form, solves the ordinary linear second-order differential equation; that is, [36]

$$
\psi^{\prime \prime}(s)+\frac{\tilde{\tau}(s)}{\sigma(s)} \psi^{\prime}(s)+\frac{\tilde{\sigma}(s)}{\sigma^{2}(s)} \psi(s)=0
$$

where the prime denotes the derivative with respect to the independent variable $s$. Here, $\sigma(s)$ and $\widetilde{\sigma}(s)$ must be polynomials of at most second degree and $\widetilde{\tau}(s)$ is a polynomial with at most first degree [36]. Using the following transformation:

$$
\psi(s)=W(s) \Phi(s),
$$

(A.1) is reduced to the well-known hypergeometric-type equation [36]

$$
\sigma(s) \Phi^{\prime \prime}(s)+\tau(s) \Phi^{\prime}(s)+\lambda \Phi(s)=0,
$$

where

$$
\tau(s)=\tilde{\tau}(s)+2 \pi(s), \quad \text { with } \tau^{\prime}(s)<0 .
$$

Equation (A.1) has a particular solution when the following relation is satisfied:

$$
\lambda=\lambda_{n}=-n \tau^{\prime}(s)-\frac{n(n-1)}{2} \sigma^{\prime \prime}(s), \quad \text { with } n=0,1,2, \ldots
$$

In order to obtain the energy eigenvalues equation, the functions given below are calculated:

$$
\begin{gathered}
\pi(s)=\frac{\sigma^{\prime}(s)-\tilde{\tau}(s)}{2} \pm \sqrt{\left(\frac{\sigma^{\prime}(s)-\tilde{\tau}(s)}{2}\right)^{2}-\tilde{\sigma}(s)+k \sigma(s),} \\
\lambda=k+\pi^{\prime}(s),
\end{gathered}
$$

where $\lambda$ is a constant. The expression under the square root in (A.6) must be the square of a polynomial of the first degree, since $\pi(s)$ is the first-degree polynomial. Then, one can obtain the $k$ values by considering that the discriminant of the square root has to be zero in (A.6) [36]. Consequently, the energy eigenvalues can be obtained by comparing (A.7) with (A.5). The function $\Phi(s)$ given in (A.3) is a hypergeometrictype function whose solution can be written in terms of the polynomials given by the Rodrigues relation

$$
\Phi_{n}(s)=\frac{C_{n}}{\rho(s)} \frac{d^{n}}{d s^{n}}\left[\sigma^{n}(s) \rho(s)\right]
$$

where $C_{n}$ is the normalization constant and the weight function $\rho(s)$ satisfies the condition

$$
[\sigma(s) \rho(s)]^{\prime}=\tau(s) \rho(s) .
$$


On the other hand, the other factor $W(s)$ satisfies the following logarithmic equation:

$$
\frac{d}{d s} \ln W(s)=\frac{\sigma(s)}{\pi(s)} .
$$

A.2. Parametric Formulation of Nikiforov-Uvarov Method. The NU method, in its parametric form, solves

$$
\begin{gathered}
\frac{d^{2}}{d s^{2}} \psi_{n}(s)+\frac{\alpha_{1}-\alpha_{2} s}{s\left(1-\alpha_{3} s\right)} \frac{d}{d s} \psi_{n}(s) \\
+\frac{-\xi_{1} s^{2}+\xi_{2} s-\xi_{3}}{\left[s\left(1-\alpha_{3} s\right)\right]^{2}} \psi_{n}(s)=0 .
\end{gathered}
$$

Here, we give only the basic ingredients of the generalized NU method. In this case, comparing (A.11) with (A.1), one can obtain

$$
\begin{gathered}
\tilde{\tau}(s)=\alpha_{1}-\alpha_{2} s, \quad \sigma(s)=s\left(1-\alpha_{3} s\right), \\
\tilde{\sigma}(s)=-\xi_{1} s^{2}+\xi_{2} s-\xi_{3} .
\end{gathered}
$$

Inserting the above equations into (A.6) leads to [37]

$$
\pi(s)=\alpha_{4}+\alpha_{5} s \pm \sqrt{\left(\alpha_{6}-k \alpha_{3}\right) s^{2}+\left(\alpha_{7}+k\right) s+\alpha_{8}}
$$

where

$$
\begin{gathered}
\alpha_{4}=\frac{1}{2}\left(1-\alpha_{1}\right), \quad \alpha_{5}=\frac{1}{2}\left(\alpha_{2}-2 \alpha_{3}\right), \\
\alpha_{6}=\alpha_{5}^{2}+\xi_{1}, \quad \alpha_{7}=2 \alpha_{4} \alpha_{5}-\xi_{2}, \\
\alpha_{8}=\alpha_{4}^{2}+\xi_{3} .
\end{gathered}
$$

Considering that the discriminant of the square root has to be zero in (A.6), we obtain [37]

$$
k_{1,2}=-\left(\alpha_{7}+2 \alpha_{3} \alpha_{8}\right) \pm 2 \sqrt{\alpha_{8} \alpha_{9}}
$$

with

$$
\alpha_{9}=\alpha_{3} \alpha_{7}+\alpha_{3}^{2} \alpha_{8}+\alpha_{6}
$$

From (A.13), one can easily see that different $k$ values lead to different $\pi(s) s$. If we take

$$
k=-\left(\alpha_{7}+2 \alpha_{3} \alpha_{8}\right)-2 \sqrt{\alpha_{8} \alpha_{9}}
$$

$\pi(s)$ becomes

$$
\pi(s)=\alpha_{4}+\alpha_{5} s-\left[\left(\sqrt{\alpha_{9}}+\alpha_{3} \sqrt{\alpha_{8}}\right) s-\sqrt{\alpha_{8}}\right],
$$

and then we find [30]

$$
\tau(s)=\alpha_{1}+2 \alpha_{4}-\left(\alpha_{2}-2 \alpha_{5}\right) s-\left[\left(\sqrt{\alpha_{9}}+\alpha_{3} \sqrt{\alpha_{8}}\right) s-\sqrt{\alpha_{8}}\right] .
$$

The energy eigenvalue equation can be readily obtained by using (A.5) and (A.6) with the above results as follows [37]:

$$
\begin{aligned}
& \alpha_{2} n-(2 n+1) \alpha_{5}+(2 n+1)\left(\sqrt{\alpha_{9}}+\alpha_{3} \sqrt{\alpha_{8}}\right) \\
& +n(n-1) \alpha_{3}+\alpha_{7}+2 \alpha_{3} \alpha_{8}+2 \sqrt{\alpha_{8} \alpha_{9}}=0 .
\end{aligned}
$$

In order to obtain the wave functions, one can use the following relations [37]:

$$
\begin{array}{r}
\rho(s)=s^{\alpha_{10}-1}\left(1-\alpha_{3} s\right)^{\left(\alpha_{11} / \alpha_{3}\right)-\alpha_{10}-1}, \\
\Phi_{n}(s)=P_{n}^{\left(\alpha_{10}-1,\left(\alpha_{11} / \alpha_{3}\right)-\alpha_{10}-1\right)}\left(1-2 \alpha_{3} s\right), \\
W(s)=s^{\alpha_{12}}\left(1-\alpha_{3} s\right)^{-\alpha_{12}-\left(\alpha_{13} / \alpha_{3}\right)}, \\
\Psi_{n}(s)=s^{\alpha_{12}}\left(1-\alpha_{3} s\right)^{-\alpha_{12}-\left(\alpha_{13} / \alpha_{3}\right)} \\
\times P_{n}^{\left(\alpha_{10}-1,\left(\alpha_{11} / \alpha_{3}\right)-\alpha_{10}-1\right)}\left(1-2 \alpha_{3} s\right),
\end{array}
$$

where $P_{n}^{\left(\alpha_{10}-1,\left(\alpha_{11} / \alpha_{3}\right)-\alpha_{10}-1\right)}\left(1-2 \alpha_{3} s\right)$ is the Jacobi polynomial and [37]

$$
\begin{gathered}
\alpha_{10}=\alpha_{1}+2 \alpha_{4}+2 \sqrt{\alpha_{8}}, \\
\alpha_{11}=\alpha_{2}-2 \alpha_{5}+2\left(\sqrt{\alpha_{9}}+\alpha_{3} \sqrt{\alpha_{8}}\right), \\
\alpha_{12}=\alpha_{4}+\sqrt{\alpha_{8}} \\
\alpha_{13}=\alpha_{5}-\left(\sqrt{\alpha_{9}}+\alpha_{3} \sqrt{\alpha_{8}}\right) .
\end{gathered}
$$

B.

A simple proof of the generalized operator form of the position momentum given in (3) is written here. Let us assume

$$
\widehat{x}_{i}=f(p) \frac{\partial}{\partial p_{i}}, \quad \widehat{p}_{i}=p_{i} .
$$

In this case, we simply have

$$
\begin{aligned}
{\left[x_{o p}, p_{o p}\right] \Psi } & =(\widehat{x} \hat{p}-\widehat{p} \hat{x}) \Psi \\
& =\left\{f(p) \frac{\partial}{\partial p} \widehat{p}-\widehat{p} f(p) \frac{\partial}{\partial p}\right\} \Psi \\
& =f(p) \frac{\partial}{\partial p}(\widehat{p} \Psi)-\widehat{p} f(p) \frac{\partial \Psi}{\partial p} \\
& =f(p) \Psi+\widehat{p} f(p) \frac{\partial \Psi}{\partial p}-\widehat{p} f(p) \frac{\partial \Psi}{\partial p}, \\
& {\left[x_{o p}, p_{o p}\right] \Psi=f(p) \Psi . }
\end{aligned}
$$

A simple comparison with (B.1) indicates the correspondence

$$
f(p)=i \hbar\left(1+\beta p^{2}\right) .
$$

\section{Acknowledgment}

H. Hassanabadi thanks Shahrood University for their support in the work.

\section{References}

[1] F. Scardigli, "Generalized uncertainty principle in quantum gravity from micro-black hole gedanken experiment," Physics Letters B, vol. 452, no. 1-2, pp. 39-44, 1999. 
[2] S. Das and E. C. Vagenas, "Universality of quantum gravity corrections," Physical Review Letters, vol. 101, no. 22, pp. 221301221305, 2008.

[3] S. Das and E. C. Vagenas, "Phenomenological implications of the generalized uncertainty principle," Canadian Journal of Physics, vol. 87, no. 3, pp. 233-240, 2009.

[4] I. Dadić, L. Jonke, and S. Meljanac, "Harmonic oscillator with minimal length uncertainty relations and ladder operators," Physical Review D, vol. 67, no. 8, pp. 87701-87705, 2003.

[5] L. N. Chang, D. Minic, N. Okamura, and T. Takeuchi, "Exact solution of the harmonic oscillator in arbitrary dimensions with minimal length uncertainty relations," Physical Review D, vol. 65, no. 12, pp. 125027-125035, 2002.

[6] S. Benczik, L. N. Chang, D. Minic, N. Okamura, S. Rayyan, and T. Takeuchi, "Short distance versus long distance physics: the classical limit of the minimal length uncertainty relation," Physical Review D, vol. 66, no. 2, Article ID 026003, 2002.

[7] K. Konishi, G. Paffuti, and P. Provero, "Minimum physical length and the generalized uncertainty principle in string theory," Physics Letters B, vol. 234, no. 3, pp. 276-284, 1990.

[8] M. Maggiore, "A generalized uncertainty principle in quantum gravity," Physics Letters B, vol. 304, no. 1-2, pp. 65-69, 1993.

[9] S. Das, E. C. Vagenas, and A. Farag Ali, "Discreteness of space from GUP II: relativistic wave equations," Physics Letters B, vol. 690, no. 4, pp. 407-412, 2010.

[10] A. F. Ali, S. Das, and E. C. Vagenas, "Proposal for testing quantum gravity in the lab," Physical Review D, vol. 84 , no. 4 , pp. 44013-44023, 2011.

[11] M. Maggiore, "Quantum groups, gravity, and the generalized uncertainty principle," Physical Review D, vol. 49, no. 10, pp. 5182-5187, 1994.

[12] M. Maggiore, "The algebraic structure of the generalized uncertainty principle," Physics Letters B, vol. 319, no. 1-3, pp. 83-86, 1993.

[13] A. Kempf, G. Mangano, and R. B. Mann, "Hilbert space representation of the minimal length uncertainty relation," Physical Review D, vol. 52, no. 2, pp. 1108-1118, 1995.

[14] J. Magueijo and L. Smolin, "String theories with deformed energy-momentum relations, and a possible nontachyonic bosonic string," Physical Review D, vol. 71, no. 2, Article ID 026010, 6 pages, 2005.

[15] J. L. Cortés and J. Gamboa, "Quantum uncertainty in doubly special relativity," Physical Review D, vol. 71, no. 6, p. 065015, 4, 2005.

[16] M. V. Battisti and G. Montani, "The big-bang singularity in the framework of a generalized uncertainty principle," Physics Letters B, vol. 656, no. 1-3, pp. 96-101, 2007.

[17] A. F. Ali and A. Tawfik, "Modified Newton's law of gravitation due to minimal length in quantum gravity," Advances in High Energy Physics, vol. 2013, Article ID 126528, 7 pages, 2013.

[18] H. Hassanabadi, S. Zarrinkamar, and E. Maghsoodi, "Scattering states of Woods-Saxon interaction in minimal length quantum mechanics," Physics Letters B, vol. 718, no. 2, pp. 657-682, 2012.

[19] L. Menculini, O. Panella, and P. Roy, "Exact solutions of the $(2+1)$ dimensional Dirac equation in a constant magnetic field in the presence of a minimal length," Physical Review D, vol. 87, no. 6, pp. 65017-65027.

[20] M. Maziashvili, "Implications of minimum-length deformed quantum mechanics for QFT/QG," Fortschritte der Physik, vol. 61, no. 6, pp. 685-700, 2013.
[21] L. J. Garay, "Quantum gravity and minimum length," International Journal of Modern Physics A, vol. 10, no. 2, pp. 145-165, 1995.

[22] W. C. Qiang and S. H. Dong, "Arbitrary l-state solutions of the rotating Morse potential through the exact quantization rule method," Physics Letters A, vol. 363, no. 3, pp. 169-176, 2007.

[23] S. H. Dong, D. Morales, and J. Garcia-Ravelo, "Exact quantization rule and its applicatrions to physical potentials," International Journal of Modern Physics E, vol. 16, no. 1, pp. 189-198.

[24] A. F. Ali, S. Das, and E. C. Vagenas, "Proposal for testing quantum gravity in the lab," Physical Review D, vol. 84, no. 4, pp. 44013-44023, 2011.

[25] S.-H. Dong, "Exact solutions of the two-dimensional Schrödinger equation with certain central potentials," International Journal of Theoretical Physics, vol. 39, no. 4, pp. 1119-1128, 2000.

[26] K. J. Oyewumi, "Analytical solutions of the kratzer-fues potential in an arbitrary number of dimensions," Foundations of Physics Letters, vol. 18, no. 1, pp. 75-84, 2005.

[27] O. Bayrak, I. Boztosun, and H. Ciftci, "Exact analytical solutions to the kratzer potential by the asymptotic iteration method," International Journal of Quantum Chemistry, vol. 107, no. 3, pp. 540-544, 2007.

[28] M. R. Setare and E. Karimi, "Algebraic approach to the Kratzer potential," Physica Scripta, vol. 75, no. 1, pp. 90-93, 2007.

[29] S. Hossenfelder, "Minimal length scale scenarios for quantum gravity," Living Reviews in Relativity, vol. 16, no. 2, 2013.

[30] D. Bouaziz and M. Bawin, "Singular inverse square potential in arbitrary dimensions with a minimal length: application to the motion of a dipole in a cosmic string background," Physical Review A, vol. 78, no. 3, pp. 32110-32118, 2008.

[31] L. N. Chang, Z. Lewis, D. Minic, and T. Takeuchi, "On the minimal length uncertainty relation and the foundations of string theory," Advances in High Energy Physics, vol. 2011, Article ID 493514, 30 pages, 2011.

[32] U. Harbach, S. Hossenfelder, M. Bleicher, and H. Stöcker, "Probing the minimal length scale by precision tests of the muon $g^{-2}$," Physics Letters B, vol. 584, no. 1-2, pp. 109-113, 2004.

[33] D. Kothawala, L. Sriramkumar, S. Shankaranarayanan, and T. Padmanabhan, "Path integral duality modified propagators in spacetimes with constant curvature," Physical Review D, vol. 80, no. 4, Article ID 044005, 2009.

[34] O. Panella, "Casimir-Polder intermolecular forces in minimal length theories," Physical Review D, vol. 76, no. 4, pp. 4501245023, 2007.

[35] P. Pedram, K. Nozari, and S. H. Taheri, "The effects of minimal length and maximal momentum on the transition rate of ultra cold neutrons in gravitational field," Journal of High Energy Physics, vol. 2011, article 93, 2011.

[36] A. F. Nikiforov and V. B. Uvarov, Special Functions of Mathematical Physics, Birkhäuser, Basel, Switzerland, 1988, 1988.

[37] C. Tezcan and R. Sever, "A general approach for the exact solution of the Schrödinger equation," International Journal of Theoretical Physics, vol. 48, no. 2, pp. 337-350, 2009. 

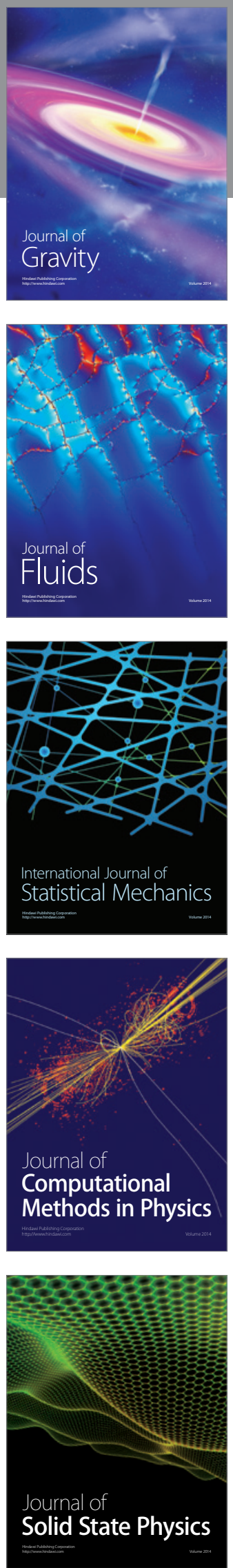

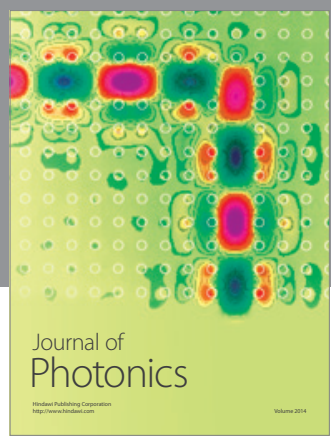

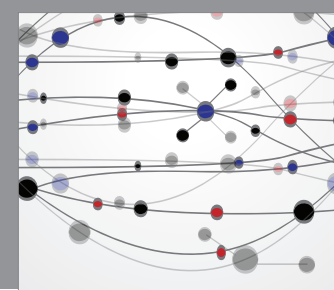

The Scientific World Journal

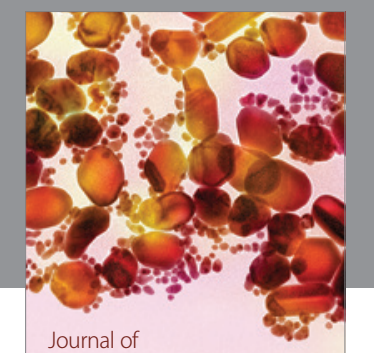

Soft Matter
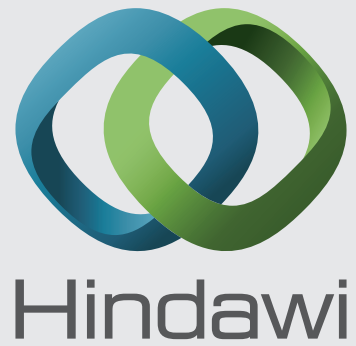

Submit your manuscripts at

http://www.hindawi.com
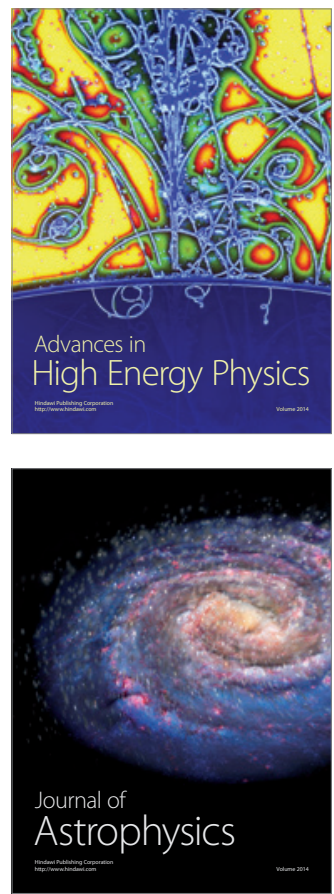
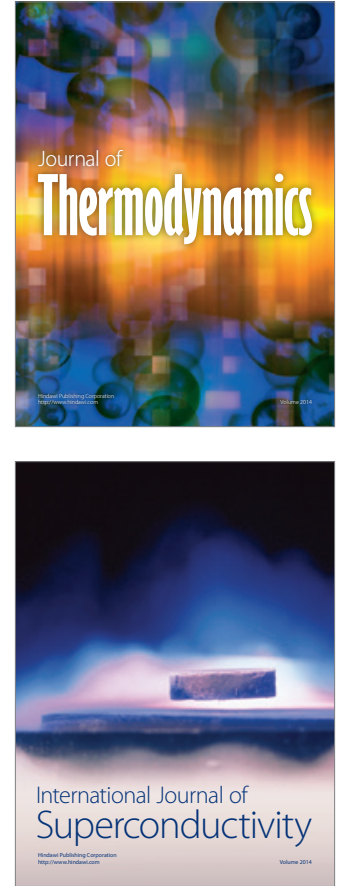
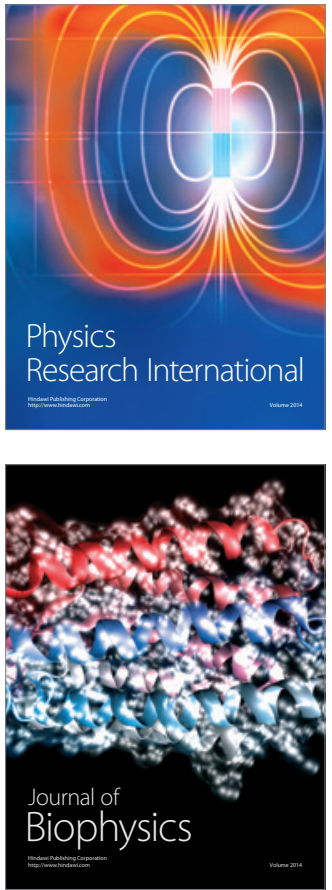
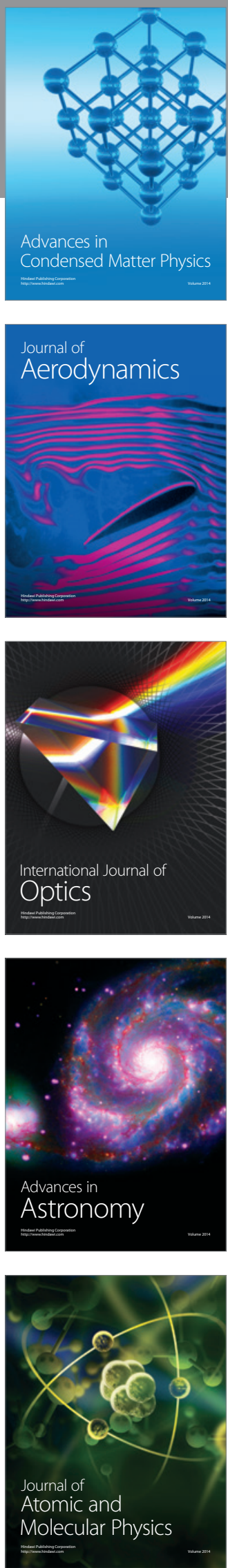